\title{
An attention-based effective neural model (1) corshath for drug-drug interactions extraction
}

\author{
Wei Zheng ${ }^{1,2}$, Hongfei Lin ${ }^{1 *}$, Ling Luo', Zhehuan Zhao', Zhengguang Li ${ }^{1,2}$, Yijia Zhang ${ }^{1}$, Zhihao Yang ${ }^{1}$ \\ and Jian Wang ${ }^{1}$
}

\begin{abstract}
Background: Drug-drug interactions (DDIs) often bring unexpected side effects. The clinical recognition of DDIs is a crucial issue for both patient safety and healthcare cost control. However, although text-mining-based systems explore various methods to classify DDls, the classification performance with regard to DDls in long and complex sentences is still unsatisfactory.

Methods: In this study, we propose an effective model that classifies DDIs from the literature by combining an attention mechanism and a recurrent neural network with long short-term memory (LSTM) units. In our approach, first, a candidate-drug-oriented input attention acting on word-embedding vectors automatically learns which words are more influential for a given drug pair. Next, the inputs merging the position- and POS-embedding vectors are passed to a bidirectional LSTM layer whose outputs at the last time step represent the high-level semantic information of the whole sentence. Finally, a softmax layer performs DDI classification.

Results: Experimental results from the DDIExtraction 2013 corpus show that our system performs the best with respect to detection and classification ( $84.0 \%$ and $77.3 \%$, respectively) compared with other state-of-the-art methods. In particular, for the Medline-2013 dataset with long and complex sentences, our F-score far exceeds those of top-ranking systems by $12.6 \%$.

Conclusions: Our approach effectively improves the performance of DDI classification tasks. Experimental analysis demonstrates that our model performs better with respect to recognizing not only close-range but also long-range patterns among words, especially for long, complex and compound sentences.
\end{abstract}

Keywords: Attention, Recurrent neural network, Long short-term memory, Drug-drug interactions, Text mining

\section{Background}

Therapy with multiple drugs is a common phenomenon in most treatment procedures. Drug-drug interactions (DDIs) occur when one administered drug influences the level or activity of another drug. DDIs often lead to unexpected side effects or a variety of adverse drug reactions (ADRs) [1]. DDIs are one of the main reasons for the majority of medical errors. Based on their financial, social and health costs, the recognition and prediction of DDIs, and hence their prevention, can greatly benefit patients and health care systems [2].

\footnotetext{
* Correspondence: hflin@dlut.edu.cn

${ }^{1}$ College of Computer Science and Technology, Dalian University of

Technology, Dalian, China

Full list of author information is available at the end of the article
}

Today, huge amounts of the most current and valuable unstructured information relevant to DDIs are hidden in specialized databases and scientific literature. Text mining based on computerized techniques may recognize patterns and discover knowledge from various available biological databases and unstructured texts [3-5]. Hence, the use of text-mining techniques to recognize DDIs from databases and texts is a promising approach. In addition, this technique contributes to the automation of the database curation process, which is currently performed manually, and the building of a biomedical knowledge graph [6].

To improve and evaluate performance with respect to classifying DDIs from biomedical texts, DDIExtraction challenges in 2011 (DDI-2011) [7] and 2013 (DDI-2013) [8] were organized successfully. Each challenge provided a benchmark corpus. DDI-2013 not only focused on the 
identification of all possible pairs of interacting drugs (the detection task) but also proposed a more finegrained classification of each true DDI (the classification task). The two tasks are regarded as binary and multiclass classification problems, respectively. In particular, the DDI-2013 corpus [9] consists of texts selected from the DrugBank database (DB-2013 dataset) and MEDLINE abstracts (ML-2013 dataset). They contain sentences with different styles. The DB-2013 dataset contains short and concise sentences, while the ML-2013 dataset usually contains long and subordinated sentences that are characterized by scientific language. Overall, the performance of existing DDI classification systems decreases drastically for long and complex sentences [10], which may be one of the main reasons for the lower F-score obtained on the ML-2013 dataset than that on the DB-2013 dataset.

Traditional studies of DDI tasks mainly use machinelearning methods such as support vector machine (SVM). In general, features such as word-level features, dependency graphs, and parser trees are designed manually by SVM-based systems [11-17], which have performed well during the past decade. Natural language processing (NLP) toolkits such as syntactic and dependency parsers are exploited to parse sentences, which unavoidably brings some unexpected errors, especially for long sentences. At present, feature extraction is still a skill-dependent task that is performed on a trial-and-error basis. In addition, it has limited lexical generalization abilities for unseen words.

By contrast, neural network (NN)-based methods are automatic representation-learning methods with multiple levels of representation, which are obtained by composing simple but non-linear modules that each transform the representation at one level into a representation at a higher, slightly more abstract level [18]. Recently, deep neural network models have shown promising results for many NLP tasks $[19,20]$. There are two main neural network architectures: convolutional neural network $(\mathrm{CNN})$ and recurrent neural network (RNN).

$\mathrm{CNN}$ with a fixed-size convolution window can capture the contextual information of a word, which is similar to the traditional n-gram feature. For the DDI-2013 tasks, these CNN-based systems [21-23] have performed well. However, the best performance (an F-score of $52.1 \%)$ on the ML-2013 dataset is not satisfactory. The semantic meaning of a drug-drug interaction in a sentence may appear in a few words before, in between, or after the candidate drug pair. The ML-2013 dataset has sentences with relatively longer and more complex structures than those of the DB-2013 dataset. Thus, some meaningful contexts that are relevant to a particular DDI are possibly non-consecutive, and there may be longer spans among them. However, the goal of CNN is to generalize local and consecutive contexts. Therefore, $\mathrm{CNN}$ is potentially weak, especially for learning longdistance patterns. CNN-based approaches that utilize multiple window sizes, dependency paths and sufficiently stacked CNN layers can solve the difficulty of CNN models in learning long-distance patterns in part. However, stacked CNN layers are generally harder to train than gated RNNs. In addition, they all either require much higher computational costs or face errors caused by a dependency parser.

By contrast, RNN with long short-term memory (LSTM) units, which is a temporal sequence model, adaptively accumulates context information of the whole sentence through memory units. Thereby, RNN is suitable for modelling long sentences without a fixed length because it has the power to learn the global and possibly non-consecutive patterns. Moreover, there are some successful RNN-based applications [17, 24, 25] for relation classification. However, words need to be transmitted one by one along the sequence in an RNN. Therefore, some important contextual information (for example, long-distance dependencies among words) could be lost in the transmission process for long texts [26].

Currently, some systems exploit the attention mechanism to address this issue. Attention-based models have shown great success in many NLP tasks such as machine translation [20, 27], question answering [28, 29] and recognizing textual entailments [30]. In the context of relation classification, the attention mechanism, weighing of text segments (e.g., word or sentence) or some highlevel feature representations obtained by learning a scoring function allows a model to pay more attention to the most influential segments of texts for a relationship category. Wang et al. [31] propose a CNN architecture based on two levels of attention for relation classification of general domains. The joint AB-LSTM model [32] combines a general pooling attention with LSTM for DDI classification. However, related experiments indicate that the introduction of pooling attention fails to improve the performance of DDI classification tasks.

In this work, with simplicity and effectiveness in mind, we extracted DDIs from biomedical texts using an attention-based neural network model called Att-BLSTM that uses RNN with LSTM units. First, a candidatedrug-oriented input attention on the representation layer was designed to automatically learn which words are more influential for a given drug pair. Next, outputs of a bidirectional LSTM at the last time step represent highlevel features of sentences. Finally, a softmax classifier conducted DDI classification. Experimental results on the DDIExtraction 2013 corpus indicate that our model yields F-score boosts of up to $2.2 \%$ and $5.8 \%$ over the current top-ranking systems for DDI detection and classification, respectively, in addition to the best F-score on 
all interaction types, especially for the Medline-2013 dataset on which our F-score outperforms the existing best result by $12.6 \%$. Our model significantly improves performance with respect to three datasets. Experiments demonstrate that our model, with an attention mechanism and fewer features, can better recognize longrange dependency patterns among words in sentences, especially in long, complex and compound sentences.

\section{Methods}

In this section, we describe the proposed network model for extracting relations of drug-drug interactions from biomedical texts in detail.

\section{Text preprocessing}

\section{Basic processing}

We first completed several common preprocessing steps on both training and test data. A special tag replaced each digit string that is not a substring of a drug entity. A bracket without either of the candidate drugs was deleted. For the generalization of our approach, all drug mentions were anonymized using $d r u g *$ (" denotes $0,1,2, \ldots$ ). Sentences of the test dataset with only one entity or two entities with the same token were filtered out because of the impossibility of a relation.

\section{Following-based anaphora}

After the DDIExtraction-2013 shared tasks, the error analysis of Segura-Bedmar et al. [10] indicates that one of the most important factors contributing to false negatives in DrugBank texts is the lack of coreference resolution. Rules in our approach were defined for some sentence patterns, including the phrase 'following [cataphora word]' with a colon, where the two entities of a candidate drug pair are on either side of the colon. We may also call this the resolution of the followingbased cataphora. In the subsequent pattern, $[w]^{*}$ denotes one or more words.

Case 1: drug1 [w]* following [cataphora word]: [w]* drug2 [w]". Replaced with: drug1 [w]" following drug2.

Case 2: [w]* following [cataphora word] [w]* drug2:[w]* drug1 $[w]^{*}$. Replaced with: $[w]^{*}$ following drug1 $[w]^{*}$ drug2.

Nevertheless, these rules do not work for the ML-2013 dataset, which has hardly any sentences with the above cases.

\section{Pruned sentences}

If there are overlong texts in a sentence, except texts between a candidate drug pair, redundant information will decrease the detection and classification performances. Therefore, we pruned each sentence to the fixed input length. After computing the maximal separation between a pair of candidate drugs, we chose an input width that is $n$ greater than the separation. Each input sentence was made of this length by either trimming longer sentences or padding shorter sentences with a special token.

\section{Network architecture}

Considering the advantages of LSTM in long-distance pattern learning, we still introduced the attention mechanism into our model to overcome the bias defect of LSTM to some extent. Figure 1 gives an overview of the network architecture. The model is composed of six layers: (1) the input layer to accept three types of information, namely, word, part of speech (POS) and relative distances between a word and each candidate drug in an input sentence; (2) the embedding layer to look up tables to encode the above input into real-valued vectors (also called embedding vectors); (3) the input attention layer to weight word-embedding vectors, which are the most influential for the relationship between a pair of special candidate drugs; (4) the merge layer to connect three corresponding embedding vectors into a vector by words; (5) a bidirectional RNN with LSTM units to learn the high-level syntactic meaning of the whole sentence and pass outputs at the last time step to the next layer; and (6) the logistic regression layer with a softmax function to perform DDI classification. The main layers will be described in detail in the following sections.

\section{Input representation}

After transforming inputs into various embedding vectors, our model feeds them to the subsequent layer. Word embedding, which was proposed by Bengio [33] (also known as distributed word representation), maps words to a low-dimensional and dense real space. It is able to capture some underlying semantic and syntactic information around words by learning from large amounts of unlabelled data. Word embedding reflects the topic similarity among words and improves their generalization to some extent.

\section{Input feature}

Given a pruned sentence $S=\left\{w_{1}, w_{2}, \ldots, w_{i}, \ldots, w_{n}\right\}$, each word $w_{i}$ is represented as three features: the word itself, part of speech and position. Each feature group has an embedding vocabulary. The position feature proposed by Zeng [34] was also introduced into our model to reflect the relative distances $\left(d_{i 1}\right.$ and $\left.d_{i 2}\right)$ between the current word $w_{i}$ and two candidate drug mentions.

In addition, the semantic meaning of a word $w_{i}$ that is reflected in a given sentence may be not necessarily consistent with its embedding vector. For example, the word "effect" is a noun as well as a verb. However, when it 


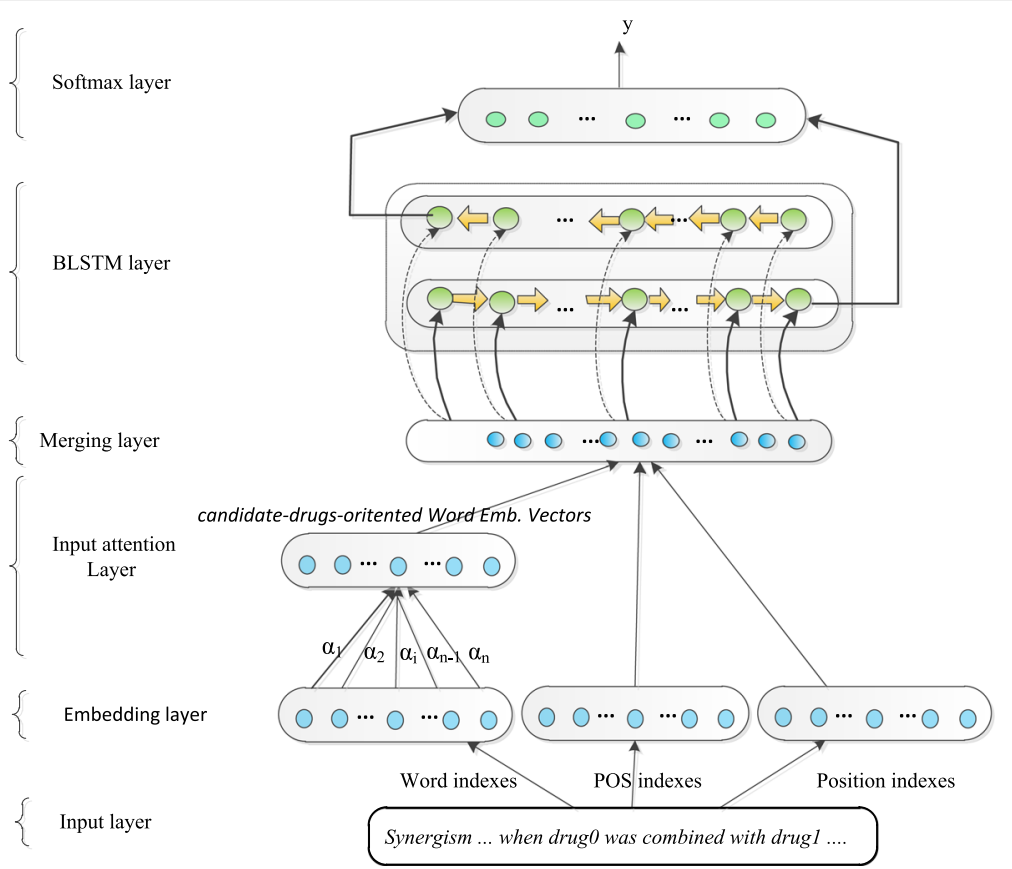

Fig. 1 The model architecture with input attention. Note: Drug0 and drug1 are the candidate drug pair

appears in different sentences with different POSs, its embedding vectors are still identical. Therefore, the POS feature is informative for DDI extraction. Our model combined a word with its POS tag (such as $\mathrm{NN}, \mathrm{VB}, \mathrm{DT}$ ) to distinguish its semantic meaning in different sentences. We obtained POS tags by using the Stanford Parser [35] to parse above processed sentences.

\section{Embedding layer}

Each feature group of the input layer has a corresponding embedding layer. Suppose $V_{k}^{l_{k} \times m_{k}}$ is the embedding vocabulary for the $k$-th $(k=1,2,3)$ feature group, where $m_{k}$ is the dimensionality (a hyper-parameter) of the embedding vector of a feature and $l_{k}$ is the number of features in the vocabulary $V_{k}$. Each embedding vocabulary can be initialized either by a random process or by some pretrained word embedding vectors. For a word $w_{i}$, the embedding layer maps the index token of each feature to a real-valued row vector by looking up its corresponding embedding vocabulary.

\section{Input attention}

Attention mechanisms have been successfully applied to sequence-to-sequence learning tasks. For relation classification tasks, attention mechanisms are able to learn a weight for each word of a sentence to reflect its level of effect on the final classification result. For a pair of candidate drugs, the DDI tasks aim to classify their relation.
It is obvious that not all words contribute equally to the sentence meaning that determines their relationship type. We expected that our model has the ability to determine which words of the sentence are the most influential for the relationship between a pair of special candidate drugs. Therefore, our model applied an attention mechanism to input word embedding for this purpose. We exploited two row vectors $\boldsymbol{\alpha}^{j}(j \in 1,2)$, whose size equals the maximum length $n$ of the sentence, to quantify the relevance degree of every word $w_{i}$ of a sentence with respect to the $j$-th drug candidate $e_{j}$.

$$
\alpha_{i}^{j}=\operatorname{soft} \max \left(\operatorname{score}\left(\boldsymbol{u}_{w_{i}}, \boldsymbol{u}_{e_{j}}\right)\right)
$$

Here, $\boldsymbol{u}_{w_{i}}$ and $\boldsymbol{u}_{e_{j}}$ are word-embedding vectors of the word $w_{i}$ and the drug candidate $e_{j}$, respectively. The score function is referred to as a candidate-drug-oriented function, for which we consider the following two alternatives: dot-score and cos-score:

$$
\begin{aligned}
& \operatorname{dot}-\operatorname{score}\left(\boldsymbol{u}_{w_{i}}, \boldsymbol{u}_{e_{j}}\right)=\frac{\operatorname{dot}\left(\boldsymbol{u}_{w_{i}}, \boldsymbol{u}_{e_{j}}\right)}{m_{1}} \quad(\mathrm{i} \leq n) \\
& \cos -\operatorname{score}\left(\boldsymbol{u}_{w_{i}}, \boldsymbol{u}_{e_{j}}\right)=\cos \left(\boldsymbol{u}_{w_{i}}, \boldsymbol{u}_{e_{j}}\right) \quad(\mathrm{i} \leq n)
\end{aligned}
$$

Here, the symbols $d o t$ and cos denote the dotproduct and cosine operations on two vectors $\boldsymbol{u}_{w_{i}}$ and $\boldsymbol{u}_{e_{j}}$, respectively. $m_{1}$ is the dimensionality of the word embedding vector. Then, the candidate-drug-oriented embedding vector $\boldsymbol{w}_{i}^{e}$ is derived from the combined 
effects of the two factors $\alpha_{i}^{1}$ and $\alpha_{i}^{2}$ acting on the original embedding vector $\boldsymbol{u}_{w_{i}}$ of the word $w_{i}$.

$$
\begin{aligned}
& \alpha_{i}=\frac{\alpha_{i}^{1}+\alpha_{i}^{2}}{2} \\
& \boldsymbol{w}_{i}^{e}=\boldsymbol{u}_{w_{i}}{ }^{*} \alpha_{i}
\end{aligned}
$$

Here, the symbol * denotes element-wise multiplication. For the sake of comparison, we still provide a noncandidate-drug-oriented function, tanh-score:

$$
\tanh -\operatorname{score}\left(\boldsymbol{S}_{w}\right)=\boldsymbol{V} \tanh \left(\boldsymbol{W} \boldsymbol{S}_{w}+\boldsymbol{b}\right)
$$

where $V$ and $W$ are learned matrices, $S_{w}$ is the embedding matrix of the sentence, and the size of the function tanh-score is same as that of the above $\boldsymbol{\alpha}^{j}$.

Finally, these vectors, including word embedding $\boldsymbol{w}_{i}^{e}$, POS embedding $\boldsymbol{w}_{i}^{p}$ and position embedding $\boldsymbol{w}_{i}^{d 1}, \boldsymbol{w}_{i}^{d 2}$ are concatenated into a new single vector $\boldsymbol{x}_{i}=\boldsymbol{w}_{i}^{e}||$ $\boldsymbol{w}_{i}^{p}|| \boldsymbol{w}_{i}^{d 1}|| \boldsymbol{w}_{i}^{d 2}$ to represent the word $w_{i}$, where $\boldsymbol{x}_{\boldsymbol{i}} \in$ $R^{m}\left(m=m_{1}+m_{2}+2 m_{3}\right)$. As a result, the sentence $S$ is a sequence of real-valued vectors $S_{\text {emb }}=\left\{\boldsymbol{x}_{1}, \boldsymbol{x}_{2}, \ldots\right.$, $\left.\boldsymbol{x}_{i}, \ldots, \boldsymbol{x}_{n}\right\}$.

\section{Recurrent neural network with long short-term memory units}

The DDI tasks are relation classifications at the sentence level. In our model, the recurrent layer plays a key role in learning both the long-range and close-range patterns among words in sequence texts.

Theoretically, an RNN [36] has the ability to process a sequence of arbitrary length by recursively applying a transition function to the internal hidden state vector of its memory unit. However, if a sequence is overlong, gradient vectors of the back-propagation algorithm tend to grow or decay exponentially [37-39] in the process of training. The LSTM network [39] was proposed by Hochreiter and Schmidhuber to specifically address this issue. LSTM introduces a separate memory cell with an adaptive gating mechanism, which determines the degree to which LSTM units maintain their previous states, and updates and exposes extracted features of the current data input. In our model, we adopted the implementation used by Graves [40].

In addition, RNN is a biased model, where later inputs are more dominant than earlier inputs if it is used to encode a sentence. For the DDI tasks, the effective features for the relation between two candidate drugs might not necessarily appear in front of the current word, and the future words may play a part in the training process of DDI classification. Therefore, our model applied a bidirectional LSTM (BLSTM). For the word $w_{i}$, the two LSTMs pick up available contextual information along the sentence forwards and backwards, which takes advantage of the previous and future context of the current term to some extent. Thus, BLSTM better captures the global semantic representation of an input sentence. Outputs $\left(\overrightarrow{\boldsymbol{h}_{n}}\right.$ and $\overleftarrow{\boldsymbol{h}_{n}}$ ) of the two LSTMs at the last time step $n$ are concatenated into a vector $\boldsymbol{h}_{n}=\overrightarrow{\boldsymbol{h}_{n}} \mid \overleftarrow{\boldsymbol{h}_{n}}$ that reflects high-level features of the whole sentence.

\section{Training and classification}

The softmax layer, a logistic regression classifier with a softmax function, classifies the relation between a pair of drugs. It takes the output $\boldsymbol{h}_{n}$ of BLSTM as its input. Its output is the probability distribution over each label type for the relation between the candidate drugs in the sentence $S$. The label with the maximum probability value is the interaction type of a candidate drug pair.

$$
\begin{aligned}
& p(y=j \mid S)=\operatorname{soft} \max \left(\boldsymbol{h}_{n} \boldsymbol{W}_{S}+\boldsymbol{b}_{S}\right) \\
& \hat{y}=\arg \max _{y \in C}(p(y=j \mid S))
\end{aligned}
$$

where the symbol $C$ is the set of DDI labels, $W_{S}$ is a learned matrix that maps the vector $\boldsymbol{h}_{n}$ linearly to the number of DDI labels, and $\boldsymbol{b}_{S}$ is a learned bias vector.

The training objective is the cross-entropy cost function, which is the negative log-likelihood of the true class label $y^{(k)}$ of each predicted sentence $S^{(k)}$ :

$$
J(\theta)=-\frac{1}{l} \sum_{k=1}^{l} \log p\left(y^{(k)} \mid S^{(k)}\right)
$$

where $l$ is the number of labelled sentences in the training set and the superscript $k$ indicates the $k$-th labelled sentence. We applied RMSprop (Resilient Mean Square Propagation) to update parameters with respect to the cost function because RMSprop is empirically an appropriate optimization algorithm for learning the $\mathrm{RNN}$ based model [41]. RMSprop does not need to adjust the learning rate manually in the process of iteration, and moreover, it has better convergence and fast convergence rate.

\section{Results and discussion \\ Datasets \\ The training and test datasets}

We trained the proposed model on the DDI-2013 corpus, including three datasets: DB-2013, ML-2013 and their union set (Overall-2013). All drug mentions and drug pairs in each sentence are annotated manually. Each drug pair is annotated as either no interaction or true interaction (the detection task), with more finegained annotations consisting of four labels: "mechanism", "effect", "advice" and "int" (the classification task). Table 1 
Table 1 Statistics for DDI- 2013 corpus

\begin{tabular}{|c|c|c|c|c|c|}
\hline \multirow[t]{2}{*}{ Instances } & \multirow[t]{2}{*}{ DDI type } & \multicolumn{2}{|l|}{ DB-2013 } & \multicolumn{2}{|l|}{ ML-2013 } \\
\hline & & Training set & Test set & Training set & Test set \\
\hline \multirow[t]{5}{*}{ Positive } & mechanism & 1257 & 278 & 62 & 24 \\
\hline & effect & 1535 & 298 & 152 & 62 \\
\hline & advice & 818 & 214 & 8 & 7 \\
\hline & int & 178 & 94 & 10 & 2 \\
\hline & Total & 3788 & 884 & 232 & 95 \\
\hline Negative & & 22,217 & 4381 & 1555 & 401 \\
\hline Total & & 26,005 & 5265 & 1787 & 434 \\
\hline
\end{tabular}

lists the statistics of this corpus. A total of 330 and 62 negative instances on the DB-2013 and ML-2013 test datasets were filtered out by the basic preprocessing stage, respectively. In particular, for the ML-2013 dataset, we trained our system on the combination of the DB-2013 and ML-2013 training datasets, as suggested by [11].

\section{The pre-training corpus of embedding vectors}

The pre-training corpus for word representations, which is approximately 2.5 gigabytes in size, consists of two parts. One part comes from all abstracts before 2016 that were obtained by querying the key word "drug" in the PubMed database, and the other one is the texts of the DDI-2013 corpus. Compared with the one-hot representation of POS tags, Zhao's experiments [23] indicate that POS representations encoded by using an auto-encoder neural network model have a better effect on the performance of a system. However, vectors encoded by an auto-encoder might contain little semantic information. Therefore, our POS training corpus contains only sentences of the DDI-2013 corpus that are labelled with POS tags (43 POS tags). The two types of embedding vectors were trained by the word2vec tool (https://code.google.com/p/word2vec/) [42]. The position embedding vectors were initialized with random values that follow the standard normal distribution.

\section{Evaluation metrics}

The performance of our system is evaluated by the standard evaluation measures (precision, recall and Fscore). F-score is defined as $F=(2 P R) /(P+R)$, where $P$ denotes the precision and $R$ denotes the recall rate. Fscore can play a balancing role between $\mathrm{P}$ and $\mathrm{R}$.

\section{Hyperparameters}

Keras library was used to implement our model. We tuned the hyperparameters of our model to optimize system performance by conducting 5 -fold sentence-level cross-validation on the training set. The choices generated by this process are listed in Table 2 .

The dimensionality of word-embedding vectors $\left(m_{1}=200\right.$ in our model) could affect system performance,
Table 2 Hyperparameters

\begin{tabular}{lll}
\hline Parameter & Parameter name & Value \\
\hline$m_{1}$ & Word emb.Size & 200 \\
$m_{2} \& m_{3}$ & POS\&position emb.Size & 10 \\
$n$ & The length of a pruned sentence & 85 \\
Mini-batch & Minimal batch & 128 \\
LSTM dim. & the number of hidden units & 230 \\
\hline
\end{tabular}

as shown in Fig. 2. On the one hand, vectors do not contain enough semantic information because of the small dimension. On the other hand, with the increase in the dimension, embedding vectors bring much more noise despite their richer semantics. Meanwhile, a system needs to spend more training time with the increasing complexity of the model. The dimensionality $m_{2}$ of the position-embedding vectors was set as 10 , as used by Zeng [34]. Figure 3 shows that our model achieves the best F-score when the maximal separation between candidate drugs is less than 75 , which contains all positive instances of three datasets. According to this result, we kept at least five extra words before and after two candidate drugs. Therefore, we set the length $n$ of a pruned sentence as 85 . We output results at each epoch. When the epoch numbers are nearly 131, 128 and 58 for the Overall-2013, DB-2013 and ML-2013 datasets, respectively, our model achieved better performance on the corresponding dataset. The number of hidden units (230) of LSTM was set as the same size of input dimension of the LSTM layer to simplify our research.

For RMSprop optimization, we set the learning rate $l r=0.001$ and the momentum item parameter $r h o=0.9$, as suggested by Tieleman et al. [41]. To alleviate the over-fitting problem, dropout [43] was applied to the LSTM and softmax layers on feed-forward networks. Dropout has improved the performance of many systems

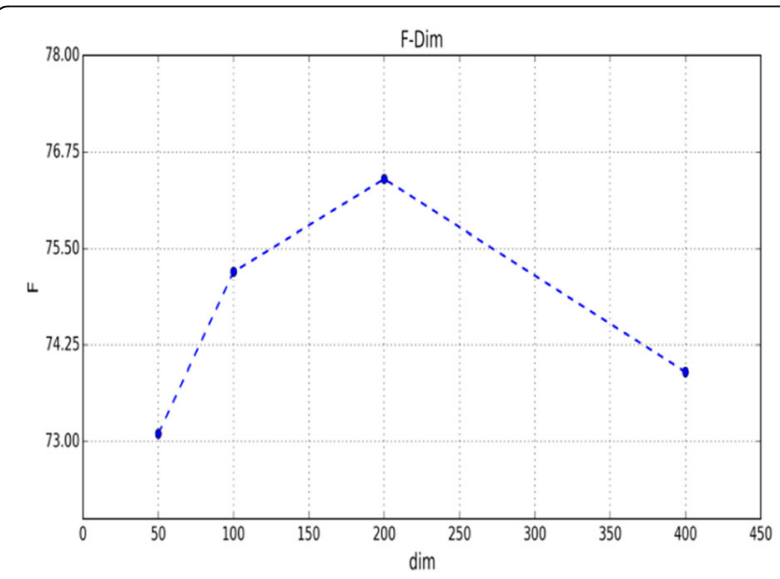

Fig. 2 Evaluation of the dimensionality of word embedding when our model without the attention mechanism was trained 


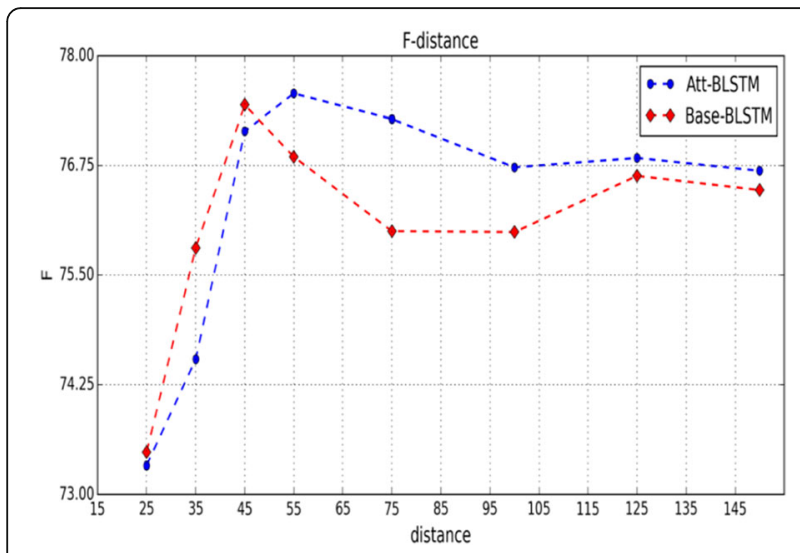

Fig. 3 F-scores of the proposed model as the distance between candidate drugs becomes longer

because it reduces the interdependency of neural units by randomly omitting feature detectors from the network during the training. The dropout rate was set to 0.5 in our model, as used by Hinton et al. [43].

\section{Effects of input attention}

We examined different score functions as described in section 2 . The results in Table 3 show that candidatedrug-oriented score functions are superior in performance to those without the special target (e.g., $\tanh$ ). The tanh-score function equivalently adds a hidden layer to the network, so performance drastically decreases with increasing complexity and noise of the model. Figure 4 shows an example for the word-level attention values calculated by dot-score used in our model, Att-BLSTM. We find that the words "synergism", "combined" and "when" have higher attention values than other words. This seems sensible in light of the ground-truth labelling as an "effect" relationship (between drugO and drug1). Hence, we might infer that the introduction of input attention highlights those influential words and makes semantic relationships between candidate drugs clear. It can be seen from Table 5 that the F-score of the model with input attention increases $23.7 \%$ over that of the model without input attention when only word embedding is considered. On the one hand, this result supports

Table 3 Performance of different score functions for the DDI classificaton on the Overall-2013 dataset

\begin{tabular}{llll}
\hline Score function & $\mathrm{P}(\%)$ & $\mathrm{R}(\%)$ & $\mathrm{F}(\%)$ \\
\hline Base_BLSTM & 74.0 & 78.6 & 76.2 \\
dot-score & 78.4 & 76.2 & 77.3 \\
cos-score & 76.3 & 76.5 & 76.4 \\
Tanh-score & 67.9 & 65.9 & 66.9 \\
\hline
\end{tabular}

Base_BLSTM is the BLSTM model without an attention mechanism which uses our all preprocessing techniques and all input embeddings including word, POS and position embedding

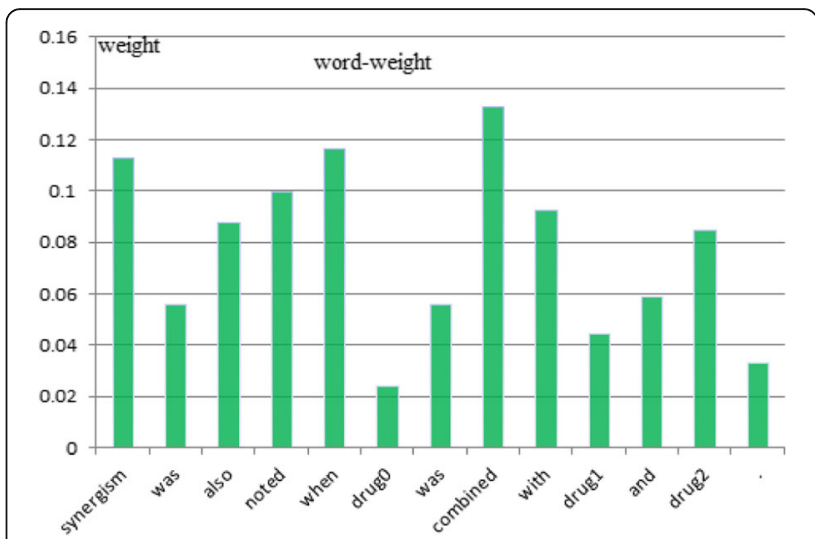

Fig. 4 Input attention visualization. Note: Drug0 and drug1 are the candidate drug pair

the above conclusion to some extent. On the other hand, it also indicates that a high F-score can be achieved by only using word embedding for the proposed model. To analyse the effect of input attention on the distance between candidate drugs, we group sentences in which the distance is lower than a fixed length in the training and test datasets. Figure 3 shows that the performance of $A t t-B L S T M$ with input attention is distinctly superior to those of Base-BLSTM without an attention mechanism when the distance is greater than 50 , whereas the Base$B L S T M$ is slightly better when the distance is less than 50 . The following reasons may explain this phenomenon. For short sentences, the Base-BLSTM model might have the ability to learn adequately from its network. Input attention equivalently appends additional restrictions to the model and requires semantic meanings to match strictly, which causes Att-BLSTM to misclassify some sentences with ambiguous semantics. However, the bias characteristic of BLSTM causes some important information to be disregarded when a sentence becomes longer. Hence, Base-BLSTM misclassifies more negative instances as positive instances as the number of positive instances increases. However, input attention in the proposed model makes up for this shortcoming. Att-BLSTM misclassifies fewer false-positive instances (fp) than Base-LSTM, despite the fact that the number of true-positive instances (tp) recognized by Att-BLSTM has slightly decreased. Table 4 lists the number of different instances detected by Base-BLSTM and Att-BLSTM on the Overall-2013 dataset. Comparing tp and fp between Base-LSTM and

Table 4 The number of different instances detected by two models for the DDI classificaton on the Overall-2013 dataset

\begin{tabular}{lllll}
\hline Model & $\mathrm{tp}$ & $\mathrm{fp}$ & $\mathrm{fn}$ & $\mathrm{tp}+\mathrm{fn}$ \\
\hline Base_BLSTM & 769 & 270 & 210 & 979 \\
Att-BLSTM & 746 & 205 & 233 & 979 \\
\hline tp
\end{tabular}

tp denotes the number of true-positive instances, fp denotes the number of false-positive instances, and $f n$ denotes the number of false-negative instances 
Att-BLSTM, the decrease in fp for Att-LSTM is nearly three times that of tp ( 65 vs. 23). Therefore, it can be seen from Table 3 that the introduction of the attention layer increases the precision and decreases the recall for the two candidate-drug-oriented attention models.

Nonetheless, input attention will not work when the distance between two drugs exceeds a threshold value. However, in practice, the distances used in our experiments should meet most of needs of relation classification at the sentence level.

\section{Effects of input representations}

We conducted experiments to evaluate the effectiveness of the strategies adopted in our method. In addition to the three embedding vectors, our model also processes the following-based cataphora and prunes sentences. Tables 5 and 6 show the effects of these steps on the performance of our model.

The position feature is an important factor that influences performance. The F-score increases by $4.7 \%$ when position embedding is introduced. Our model with the position feature further intensifies the significant contextual combination by distinguishing semantic meanings from the current word and drug entities. Moreover, the proposed model further improves the Fscore when POS embedding is incorporated. Furthermore, it can be seen from Table 6 that some preprocessing of the given sentences effectively improves the performance of our system. However, our model also performs well even if texts are not pre-processed.

\section{Performance comparisons with other systems}

To evaluate our approach, we compared our system with top-ranking systems in the DDI tasks.

\section{Other systems for comparison}

For the DDI tasks, most existing systems are based on either SVM or NN. We compare the performance of the proposed model with those of the following baseline

Table 5 Performance changes with different input representations on the overall-2013 dataset

\begin{tabular}{llll}
\hline Input representation & $\mathrm{P}(\%)$ & $\mathrm{R}(\%)$ & $\mathrm{F}(\%)$ \\
\hline (1): word without attention & 54.7 & 42.8 & 48.0 \\
(2): word + att & 76.5 & 67.5 & 71.7 \\
(3): word + att + pos & 70.9 & 74.7 & 72.7 \\
(4): word + att + position & 79.1 & 73.9 & 76.4 \\
(5): word + att + pos + position & 78.4 & 76.2 & 77.3 \\
\hline
\end{tabular}

Every model in this table uses all preprocessing techniques of our approach. Word without attention denotes the model without the attention mechanism which uses only word embedding. Word + att denotes the model which uses the attention mechanism and word embedding
Table 6 Performance changes with different preprocessing procedures on the overall-2013 dataset

\begin{tabular}{llll}
\hline Processing procedure & $\mathrm{P}(\%)$ & $\mathrm{R}(\%)$ & $\mathrm{F}(\%)$ \\
\hline (1): only candidate drugs replaced & 75.9 & 68.7 & 71.5 \\
(2): basic processing & 77.5 & 72.3 & 74.8 \\
(3): (2) + Following anaphora & 76.9 & 76.5 & 76.7 \\
(4): (3) + Pruned Sentences & 78.4 & 76.2 & 77.3 \\
\hline
\end{tabular}

Every model in this table uses three input embeddings of our approach

methods. All compared systems have performs well and have their own pros and cons.

(1)SVM-based methods: SVM-based methods commonly depend either on carefully handcrafted features or on elaborately designed kernels, which replace the dot product with a similarity function between two vectors. RAIHANI [17] designs many rules and features such as chunk, trigger words, filtering negative sentence and SAME_BLOK. This system still designs different features for the SVM classifier of each subtype. FBK-irst [11] combines different characteristics of three kernels. UTurku [14] uses informatics from domain resources such as DrugBank, in addition to sentence and dependency features.

(2)NN-based methods: NN-based methods learn the high-level representation of a sentence by the CNN or LSTM architecture. For CNN-based systems, MCCNN [21] uses multichannel word embedding vectors and SCNN [23] combines traditional features and embedding-based convolutional features. For LSTM-based methods, joint AB-LSTM combines two LSTM networks, one of which exploits the pooling attention. For the above methods, word embedding is an indispensable feature; position embedding is used by all of them except MCCNN, and some filtering techniques are exploited to rule out irrelevant negative instances, in addition to common preprocessing techniques for texts.

\section{Overall performance}

Table 7 shows that our model achieves the best performance on the Overall-2013 test dataset for both DDI detection (DEC) and DDI classification (CLA). Our Fscores for the two tasks are $2.2 \%$ and $5.8 \%$ higher than those of current best results, respectively. In addition, we observe from Table 8 that our Att-BLSTM has the characteristics of both higher precision and higher recall on the three datasets, while most existing systems have relatively lower recall values. To give every model a fair comparison, Table 9 lists performance of NN-based systems on the overall-2013 dataset for DDI classification if 
Table 7 Performance comparisons (F-score) with top-ranking systems on the overall-2013 dataset for DDI detection and DDI classification

\begin{tabular}{llllllll}
\hline Method & Team & CLA & DEC & MEC & EFF & ADV & INT \\
\hline SVM & RAlHANI [17] & $\mathbf{7 1 . 1}$ & 81.5 & $\mathbf{7 3 . 6}$ & 69.6 & $\mathbf{7 7 . 4}$ & $\mathbf{5 2 . 4}$ \\
& Context-Vector [15] & 68.4 & $\mathbf{8 1 . 8}$ & 66.9 & $\mathbf{7 1 . 3}$ & 71.4 & 51.6 \\
& Kim [16] & 67.0 & 77.5 & 69.3 & 66.2 & 72.5 & 48.3 \\
& FBK-irst [11] & 65.1 & 80.0 & 67.9 & 62.8 & 69.2 & 54.7 \\
& WBI [12] & 60.9 & 75.9 & 61.8 & 61.0 & 63.2 & 51.0 \\
& UTurku [14] & 59.4 & 69.9 & 58.2 & 60.0 & 63.0 & 50.7 \\
NN & joint AB-LSTM [32] & $\mathbf{7 1 . 5}$ & $\mathbf{8 0 . 3}$ & $\mathbf{7 6 . 3}$ & 67.6 & $\mathbf{7 9 . 4}$ & 43.1 \\
& MCCNN [21] & 70.2 & $\mathbf{7 9 . 0}$ & $\mathbf{7 2 . 2}$ & 68.2 & 78.2 & $\mathbf{5 1 . 0}$ \\
& Liu CNN [22] & 69.8 & - & 70.2 & $\mathbf{6 9 . 3}$ & 77.8 & 48.4 \\
& Zhao SCNN [23] & 68.6 & $\mathbf{7 7 . 2}$ & - & - & - & - \\
Ours & Att-BLSTM & $\mathbf{7 7 . 3}$ & $\mathbf{8 4 . 0}$ & $\mathbf{7 7 . 5}$ & $\mathbf{7 6 . 6}$ & $\mathbf{8 5 . 1}$ & $\mathbf{5 7 . 7}$
\end{tabular}

The listed results come from the corresponding papers. The symbol "_" denotes no corresponding values, because the related paper did not provide complete results (similarly hereinafter). " $D E C$ " only indicates DDI detection. "CLA" indicates DDI classification. "MEC", "EFF", "ADV" and "INT" denote "mechanism", "effect", "advice" and "int" types, respectively. The highest scores are highlighted in bold

systems don't use main text processing techniques. Our model only replaces the candidate drugs (row (1) in Table 6), while other systems use basic text processing and replaced candidate drugs (negative instances aren't filtered). We don't provide comparisons with SVMbased systems. One of the reasons is that these systems have the different kind of architecture with ours. Moreover, most systems didn't provide their source codes, and their papers didn't present results of text preprocessing on the overall-2013 dataset either.

\section{Performance on interaction types}

In addition, as far as the performance of all four interaction types are concerned, Table 7 shows that
Att-BLSTM far surpasses other systems. The four interaction types in descending order of the degree of classification difficulty are "int", "effect", "mechanism" and "advice". The performance of each subtype is shown in Table 10. Compared with the CNN-based and other LSTM-based systems, Att-BLSTM achieves obvious increases in F-score of $6.7 \%$ and $14.6 \%$ on the "int" type with the fewest training and test instances and of $8.4 \%$ and $9.0 \%$ on the "effect" type with high semantic complexities, respectively. Moreover, our F-scores on the "mechanism" and "advice" types show more than $1.2 \%$ and $5.7 \%$ relative improvements compared with the best values, respectively.

\section{Performance on the ML-2013 and DB-2013 datasets}

To compare the performance on different types of documents, the results from the ML-2013 and DB-2013 datasets are shown in Table 8 . It should be noted that our performance represents a significant improvement on the ML-2013 dataset for DDI classification. Our Fscore exceeds those of the best existing systems by more than $12.6 \%$. Meanwhile, our F-score on the DB2013 dataset outperforms those of the best NN-based and SVM-based systems by more than $5.8 \%$ and 3.3\%, respectively.

\section{Performance analysis}

The associated contexts of a word might have a longer span due to the characteristics of the DDI-2013 corpus. Therefore, it is especially important to capture relations among long-range words. However, some global and possibly non-consecutive patterns cannot be adequately learned by CNN-based and SVM-based systems. Although RAIHANI [17] has the best F-score of the SVM-based systems, this system depends too much on NLP toolkits and manual intervention. For CNN-based systems [21-23], Zhang's experiments [24] demonstrate

Table 8 Performance comparisons (F-score) with top-ranking systems on the three datasets

\begin{tabular}{|c|c|c|c|c|c|c|c|c|c|c|}
\hline \multirow[t]{2}{*}{ Method } & \multirow[t]{2}{*}{ Team } & \multicolumn{3}{|c|}{ DB-2013 } & \multicolumn{3}{|c|}{ ML-2013 } & \multicolumn{3}{|c|}{ Overall-2013 } \\
\hline & & $P(\%)$ & $R(\%)$ & $F(\%)$ & $\mathrm{P}(\%)$ & $R(\%)$ & $F(\%)$ & $\mathrm{P}(\%)$ & $R(\%)$ & $F(\%)$ \\
\hline \multirow[t]{6}{*}{ SVM } & RAIHANI [17] & - & - & 74.0 & - & - & 43.0 & 73.7 & 68.7 & 71.1 \\
\hline & Context-Vector [15] & - & - & 72.4 & - & - & 52.0 & - & - & 68.4 \\
\hline & Kim [16] & - & - & 69.8 & - & - & 38.2 & - & - & 67.0 \\
\hline & FBK-irst [11] & 66.7 & 68.6 & 67.6 & 41.9 & 37.9 & 39.8 & 64.6 & 65.6 & 65.1 \\
\hline & WBI [12] & 65.7 & 60.9 & 63.2 & 45.3 & 30.5 & 36.5 & 64.2 & 57.9 & 60.9 \\
\hline & UTurku [14] & 73.8 & 53.5 & 62.0 & 59.3 & 16.8 & 26.2 & 73.2 & 49.9 & 59.4 \\
\hline \multirow[t]{3}{*}{ NN } & MCCNN [21] & - & - & 70.8 & - & - & - & 76.0 & 65.3 & 70.2 \\
\hline & Liu CNN [22] & 77.0 & 66.7 & 71.5 & 61.4 & 45.3 & 52.1 & 75.7 & 64.7 & 69.8 \\
\hline & Zhao SCNN [23] & 73.6 & 67.0 & 70.2 & 39.4 & 39.1 & 39.2 & 72.5 & 65.1 & 68.6 \\
\hline Ours & Att-BLSTM & 78.9 & 75.7 & 77.3 & 71.8 & 59.0 & 64.7 & 78.4 & 76.2 & 77.3 \\
\hline
\end{tabular}

The highest scores are highlighted in bold 
Table 9 Performance comparisons (F-score) with NN-based systems on the overall-2013 dataset for DDI classification if systems don't use main processing techniques

\begin{tabular}{lllll}
\hline Method & Team & $\mathrm{P}(\%)$ & $\mathrm{R}(\%)$ & $\mathrm{F}(\%)$ \\
\hline NN-based & joint AB-LSTM [32] & 71.3 & 66.9 & 69.3 \\
& MCCNN [21] & - & - & 67.8 \\
& Liu CNN [22] & 75.3 & 60.4 & 67.0 \\
& Zhao SCNN [23] & 68.5 & 61.0 & 64.5 \\
Our model & Att-BLSTM & $\mathbf{7 5 . 9}$ & $\mathbf{6 8 . 7}$ & $\mathbf{7 1 . 5}$
\end{tabular}

The listed results come from the corresponding papers. The symbol "-" denotes no corresponding values, because the related paper did not provide complete results. Our model only replaces the candidate drugs (row (1) in Table 6), while other systems use basic text processing and replaced candidate drugs (negative instances aren't filtered). The highest scores are highlighted in bold

that $\mathrm{CNN}$ splits the semantic meaning into separate word segments and mixes them together so that it learns only local patterns for classification tasks.

By contrast, our approach has the ability to identify the dependency patterns of long-distance words. There are two main reasons for this. One reason is to exploit the characteristic of LSTM that adaptively accumulates contextual information word by word. The semantic meaning (the output at the last time step) of our BLSTM layer with fitted input features is actually based on contributions of all words in a sentence. Thus, our model can adequately capture the integrated contextual information. Experiments [24] indicate that RNN-based systems are similar to CNN-based systems in performance when the distance among interdependent words has a relatively small span; otherwise, RNN has clear advantages over CNN. In this respect, the joint AB-LSTM model [32] uses the same BLSTM as ours.

The other reason for the significant improvement in our performance may be the contribution of input attention that targets the candidate drugs. It provides more targeted semantic matching and causes our model to explicitly find important cue words. Consequently, the introduction of input attention further enhances the memory of LSTM of influential segments for classifying the relation between a long-distance drug pair. Therefore, our model is able to classify DDI types effectively (see Fig. 3). Finally, the above two reasons also lead to a large margin of performance improvement on the ML2013 dataset with sentences of more complex

Table 10 Performance of interaction types on the overall-2013 dataset

\begin{tabular}{llll}
\hline Subtype & $P(\%)$ & $R(\%)$ & $F(\%)$ \\
\hline EFF & 71.9 & 81.9 & 76.6 \\
MEC & 84.1 & 71.9 & 77.5 \\
ADV & 84.8 & 85.5 & 85.1 \\
INT & 75.0 & 46.9 & 57.7 \\
\hline
\end{tabular}

subordinated structures. In the case of the attention mechanism, although the joint AB-LSTM model [32] also intends to exploit the attention mechanism to capture the important clues for DDI classification, their experiments demonstrate that the introduced attentive pooling degrades rather than increases their F-score. The reason for this finding may be that their introduced attentive pooling is a non-targeted general approach rather than a target-specific approach. Moreover, their system has higher time and model complexities compared with our system because of the combination of two LSTM models.

Furthermore, although the use of techniques to filter negative instances by most of the systems partly balances the biased dataset, many positive instances are removed from the training set, which causes their model to lose many learning opportunities.

\section{Conclusion}

In this study, we applied a neural network model, AttBLSTM, based on RNN with LSTM units and an attention mechanism for classifying DDIs from biomedical texts. By introducing input attention, our model overcomes the bias deficiency of LSTM to some extent, which omits some important previous information when processing long sentences. The proposed model only depends on three input embedding vectors and the simple network architecture. Our model achieves a good overall performance on the detection and classification tasks for the DDI-2013 corpus. In particular, our Fscore far outperforms the current best F-score by $12.6 \%$ on the ML-2013 dataset, which indicates the effectiveness of our approach. Experimental analysis indicates that our approach can effectively recognize not only close-range but also long-range patterns among words in long and complex sentences.

\section{Acknowledgements}

We would like to thank the editors and all anonymous reviewers for valuable suggestions and constructive comments. We would like to thank the Natural Science Foundation of China.

\section{Funding}

This work was supported by the Natural Science Foundation of China (No.61572102 and No.61272004) and the Major State Research Development Program of China (No2016YFC0901900). The funding bodies did not play any role in the design of the study, data collection and analysis, or preparation of the manuscript.

\section{Availability of data and materials}

The datasets generated and analysed during the current study are available from the corresponding author on reasonable request.

\section{Authors' contributions}

WZ carried out the overall algorithm design and experiments. $\mathrm{HL}, \mathrm{LL}$ and ZZ participated in the algorithm design. ZL, YZ, ZY and JW participated in experiments. All authors participated in manuscript preparation. All authors read and approved the final manuscript. 


\section{Ethics approval and consent to participate}

Not applicable.

\section{Consent for publication}

Not applicable.

\section{Competing interests}

The authors declare that they have no competing interests.

\section{Publisher's Note}

Springer Nature remains neutral with regard to jurisdictional claims in published maps and institutional affiliations.

\section{Author details}

${ }^{1}$ College of Computer Science and Technology, Dalian University of Technology, Dalian, China. ${ }^{2}$ College of Software, Dalian JiaoTong University, Dalian, China.

\section{Received: 28 April 2017 Accepted: 2 October 2017}

\section{Published online: 10 October 2017}

\section{References}

1. Miranda V, Fede A, Nobuo M, Ayres V, Giglio A, Miranda M, Riechelmann RP. Adverse Drug Reactions and Drug Interactions as Causes of Hospital Admission in Oncology. J Pain Symptom Manage. 2011;42(3):342-53.

2. Agrawal A. Medication errors: prevention using information technology systems. Br J Clin Pharmacol. 2009;67(6):681.

3. Safdari R, Ferdousi R, Aziziheris K, Niakankalhori SR, Omidi Y. Computerized techniques pave the way for drug-drug interaction prediction and interpretation. Bioimpacts. 2016;6(2):71-8.

4. Ananiadou S, Kell DB, Tsujii JI. Text mining and its potential applications in systems biology. Trends Biotechnol. 2006;24(12):571-9.

5. Ben Abacha A, Chowdhury MFM, Karanasiou A, Mrabet $Y$, Lavelli A, Zweigenbaum P. Text mining for pharmacovigilance: Using machine learning for drug name recognition and drug-drug interaction extraction and classification. J Biomed Inform. 2015;58:122-32.

6. Percha B, Altman RB. Informatics confronts drug-drug interactions. Trends Pharmacol Sci. 2013;34(3):178-84.

7. Segura-Bedmar I, Martınez P, Sánchez-Cisneros D. The 1st DDIExtraction2011 challenge task: Extraction of Drug-Drug Interactions from biomedical texts. In: Proceedings of the 1st Challenge Task on Drug-Drug Interaction Extraction, vol. 761; 2011. p. 1-9.

8. Segura Bedmar I, Martínez P, Herrero Zazo M. Semeval-2013 task 9: Extraction of drug-drug interactions from biomedical texts (ddiextraction 2013). In: Seventh International Workshop on Semantic Evaluation (SemEval 2013): 2013; 2013

9. Herrerozazo M, Segurabedmar I, Declerck T. The DDI corpus: an annotated corpus with pharmacological substances and drug-drug interactions. J Biomed Inform. 2013;46(5):914-20.

10. Segura-Bedmar I, Martínez P, Herrero-Zazo M. Lessons learnt from the DDIExtraction-2013 shared task. J Biomed Inform. 2014;51:152-64.

11. Chowdhury MFM, Lavelli A. FBK-irst: A multi-phase kernel based approach for drug-drug interaction detection and classification that exploits linguistic information. In: Seventh International Workshop on Semantic Evaluation (SemEval 2013), Second Joint Conference on Lexical and Computationa Semantics (* SEM); 2013. p. 351-5.

12. Thomas $P$, Neves $M$, Rocktäschel $T$, Leser U, WBI-DDI: drug-drug interaction extraction using majority voting. In: Seventh International Workshop on Semantic Evaluation (SemEval 2013); 2013. p. 628-35.

13. Bobic T, Fluck J, Hofmann-Apitius M. SCAl: Extracting drug-drug interactions using a rich feature vector. In: Seventh International Workshop on Semantic Evaluation (SemEval 2013); 2013. p. 675-83.

14. Björne J, Kaewphan S, Salakoski T. UTurku: drug named entity recognition and drug-drug interaction extraction using SVM classification and domain knowledge.In: Seventh International Workshop on Semantic Evaluation (SemEval 2013); 2013. p. 651-59.

15. Zheng W, Lin H, Zhao Z, Xu B, Zhang Y, Yang Z, Wang J. A graph kernel based on context vectors for extracting drug-drug interactions. J Biomed Inform. 2016;61:34-43.
16. Kim S, Liu H, Yeganova L, Wilbur WJ. Extracting drug-drug interactions from literature using a rich feature-based linear kernel approach. J Biomed Inform. 2015;55:23-30.

17. Raihani A, Laachfoubi N. EXTRACTING DRUG-DRUG INTERACTIONS FROM BIOMEDICAL TEXT USING A FEATURE-BASED KERNEL APPROACH. J Theor Appl Inf Technol. 2016;92(1):109.

18. LeCun Y, Bengio Y, Hinton G. Deep learning. Nature. 2015;521(7553):436-44.

19. Li F, Zhang Y, Zhang M, Ji D. Joint Models for Extracting Adverse Drug Events from Biomedical Text. In: IJCAl; 2016. p. 2838-44.

20. Luong M-T, Pham H, Manning CD. Effective approaches to attention-based neural machine translation. In: arXiv preprint arXiv:150804025; 2015.

21. Quan C, Hua L, Sun X, Bai W. Multichannel Convolutional Neural Network for Biological Relation Extraction. Biomed Res Int. 2016;2016(2-1):1-10.

22. Liu S, Tang B, Chen Q, Wang X. Drug-drug interaction extraction via convolutional neural networks. Comput Math Methods Med. 2016:2016:1-8.

23. Zhao Z, Yang Z, Luo L, Lin H, Wang J. Drug drug interaction extraction from biomedical literature using syntax convolutional neural network. Bioinformatics. 2016;32(22):3444-53.

24. Zhang D, Wang D. Relation Classification via Recurrent Neural Network. In: arXiv preprint arXiv:150801006; 2015

25. Li F, Zhang M, Fu G, Ji D. A neural joint model for entity and relation extraction from biomedical text. BMC Bioinformatics. 2017:18(1):198.

26. Liu P, Qiu X, Chen X, Wu S, Huang X. Multi-Timescale Long Short-Term Memory Neural Network for Modelling Sentences and Documents. In: Conference on Empirical Methods in Natural Language Processing (EMNLP); 2015. p. 2326-35.

27. Bahdanau D, Cho K, Bengio Y. Neural machine translation by jointly learning to align and translate. In: arXiv preprint arXiv:14090473; 2014

28. dos Santos CN, Tan M, Xiang B, Zhou B. Attentive pooling networks. In: CoRR, abs/160203609; 2016.

29. Weston J, Chopra S, Bordes A. Memory networks. In: arXiv preprint arXiv: 14103916; 2014

30. Rocktäschel T, Grefenstette E, Hermann KM, Kočiský T, Blunsom P. Reasoning about entailment with neural attention. In: arXiv preprint arXiv:150906664; 2015.

31. Wang L, Cao Z, de Melo G, Liu Z. Relation classification via multi-level attention cnns. In: Proceedings of the 54th Annual Meeting of the Association for Computational Linguistics Association for Computational Linguistics; 2016.

32. Sahu SK, Anand A. Drug-Drug Interaction Extraction from Biomedical Text Using Long Short Term Memory Network. In: arXiv preprint arXiv:170108303; 2017.

33. Bengio $Y$, Ducharme $R$, Vincent $P$, Jauvin $C$. A neural probabilistic language model. J Mach Learn Res. 2003;3(Feb):1137-55.

34. Zeng D, Liu K, Lai S, Zhou G, Zhao J: Relation classification via convolutional deep neural network. 2014

35. De Marneffe M-C, MacCartney B, Manning CD. Generating typed dependency parses from phrase structure parses. In: Proceedings of LREC; 2006. p. 449-54

36. Elman JL. Finding structure in time. Cogn Sci. 1990:14(2):179-211.

37. Bengio $Y$, Simard $P$, Frasconi $P$. Learning long-term dependencies with gradient descent is difficult. IEEE Trans Neural Netw. 1994:5(2):157-66.

38. Hochreiter S, Bengio Y, Frasconi P, Schmidhuber J. Gradient flow in recurrent nets: the difficulty of learning long-term dependencies. In: A field guide to dynamical recurrent neural networks: Wiley-IEEE Press; 2001:237-43.

39. Hochreiter S, Schmidhuber J. Long short-term memory. Neural Comput. 1997:9(8):1735-80.

40. Graves A. Generating sequences with recurrent neural networks. In: arXiv preprint arXiv:13080850; 2013.

41. Tieleman T, Hinton G. Lecture 6.5-rmsprop: Divide the gradient by a running average of its recent magnitude. COURSERA: Neural Netw Mach Learn. 2012;4(2)

42. Mikolov T, Sutskever I, Chen K, Corrado G, Dean J. Distributed Representations of Words and Phrases and their Compositionality. In: International Conference on Neural Information Processing Systems; 2013:3111-19.

43. Hinton GE, Srivastava N, Krizhevsky A, Sutskever I, Salakhutdinov RR. Improving neural networks by preventing co-adaptation of feature detectors. In: arXiv preprint arXiv:12070580; 2012 